\title{
Supergravity, nonconformal field theories, and brane-worlds
}

\author{
Tony Gherghetta \\ Institute of Theoretical Physics, University of Lausanne, CH-1015 Lausanne, Switzerland \\ Yaron $\mathrm{Oz}$ \\ Theory Division, CERN, CH-1211 Geneva 23, Switzerland \\ (Received 4 July 2001; published 24 January 2002)
}

\begin{abstract}
We consider the supergravity dual descriptions of nonconformal super Yang-Mills theories realized on the world-volume of $\mathrm{D} p$-branes. We use the dual description to compute stress-energy tensor and current correlators. We apply the results to the study of dilatonic brane-worlds described by nonconformal field theories coupled to gravity. We find that brane-worlds based on D4- and D5-branes exhibit a localization of gauge and gravitational fields. We calculate the corrections to the Newton and Coulomb laws in these theories.
\end{abstract}

DOI: 10.1103/PhysRevD.65.046001

\section{INTRODUCTION}

The AdS/CFT correspondence relates a conformal field theory in $d$ dimensions to string theory on $\mathrm{AdS}_{d+1}$ space [1-3]. (For a review see Ref. [4].) The radial coordinate of $\operatorname{AdS}_{d+1}$, often denoted by $U$, is interpreted as an energy scale from the point of view of the $d$-dimensional conformal field theory. The canonical example is the $\mathcal{N}=4$ superconformal field theory (SCFT) in four dimensions realized on the world volume of D3-branes. The dual description is a type-IIB string theory on $\mathrm{AdS}_{5} \times \mathrm{S}^{5}$. The worldvolume theory of $\mathrm{D} p$-branes when $p \neq 3$ is a nonconformal super Yang-Mills (SYM) theory with sixteen supercharges. These theories have dual descriptions that vary as we move in the energy scale $U[5]$. They have been much less studied compared to the conformal field theories.

Another line of research is the study of brane-world scenarios $[6,7]$. We will consider a brane-world to be a brane (not necessarily a $\mathrm{D} p$-brane) located at $U=U_{*}$. When $U_{*}$ is infinite and the brane is a $\mathrm{D} p$-brane we recover the above, where the $\mathrm{D} p$-brane theory is a Yang-Mills field theory without gravity. When $U_{*}$ is finite, the theory on the brane is a Yang-Mills theory coupled to gravity with a UV cutoff $\Lambda$ $=U_{*}$. As in the duality between gauge theory and gravity, the brane-world case with $p=3$ has been extensively studied as a conformal field theory coupled to a gravity system. This brane-world scenario exhibits a localization of gravity on the brane-world. However, a bulk gauge field is not localized in this scenario.

In this paper we will consider brane-worlds based on $\mathrm{D} p$-branes when $p \neq 3$. We will start by considering the supergravity dual descriptions and use it to compute the stressenergy tensor and current correlators of the nonconformal field theories. We will then apply the results to the study of dilatonic brane-worlds described by nonconformal field theories coupled to gravity. We will find that brane-worlds based on D4- and D5-branes exhibit a localization of gauge and gravitational fields, and we calculate the corrections to the Newton and Coulomb laws in these theories.

The paper is organized as follows. In Sec. II we will consider the worldvolume theory of $N$ coincident $\mathrm{D} p$-branes in type-II string theory using the dual supergravity description.
We will compute correlation functions of the stress-energy tensor and global symmetry currents. In Sec. III we will consider brane-world scenarios with a brane located at $U$ $=U_{*}$. When $p \neq 3$ the theory of the brane-world is dual to a $(p+1)$-dimensional nonconformal super Yang-Mills theory coupled to gravity with a cutoff $\Lambda=U_{*}$. These brane-world scenarios are sometimes called dilatonic domain walls. We will analyze these brane-world scenarios with and without additional compactification of brane worldvolume coordinates. We will use the results of Sec. II in order to compute the corrections to Newton and Coulomb laws. We will also derive these corrections in a slightly different approach and discuss the localization of gauge and gravitational fields. We find that brane-worlds based on D4- and D5-branes exhibit a localization of both gauge and gravitational fields. Section IV is devoted to a more detailed discussion on brane-world scenarios based on dilatonic domain walls. Some details of Green's functions computations are outlined in the Appendix.

\section{D $p$-BRANES}

In this section we will consider the worldvolume theory of $N$ coincident $\mathrm{D} p$-branes in type-II string theory using the dual supergravity description. We will compute correlation functions of the stress-energy tensor and global symmetry currents.

\section{A. The dual supergravity description}

Consider a system of $N$ coinciding flat $\mathrm{D} p$-branes of type-II string theory. We denote the ten-dimensional space coordinates by $x_{0}, \ldots, x_{9}$. The $p+1$ worldvolume coordinates are taken to be $x_{0}, \ldots, x_{p}$. The field theory limit of this $\mathrm{D} p$-brane system is defined by taking the string length $l_{s} \rightarrow 0$ while keeping the Yang-Mills coupling $g_{Y M}^{2}=g_{s} l_{s}^{p-3}$ fixed, where $g_{s}$ is the string coupling [5]. For $p<6$ this is a limit that decouples the open string degrees of freedom attached to the $\mathrm{D} p$-branes worldvolume from the bulk closed string degrees of freedom. The worldvolume theory is a ( $p$ +1 )-dimensional $U(N)$ super Yang-Mills theory with six- 
teen supercharges. In the following we will consider the large $N$ 't Hooft limit, where we denote the 't Hooft parameter by $\lambda=g_{Y M}^{2} N$.

This system has a conjectured dual string description [5]. In the supergravity approximation the (string-frame) metric describing the system is given by

$$
\begin{aligned}
d s^{2}= & l_{s}^{2}\left(\frac{U^{(7-p) / 2}}{\lambda^{1 / 2}} d x_{\|}^{2}+\frac{\lambda^{1 / 2}}{U^{(7-p) / 2}} d U^{2}\right. \\
& \left.+\lambda^{1 / 2} U^{(p-3) / 2} d \Omega_{8-p}^{2}\right), \\
e^{\phi} & =\frac{\lambda^{(7-p) / 4}}{N} U^{(7-p)(p-3) / 4} .
\end{aligned}
$$

Note that we have omitted numerical factors that will be irrelevant for our discussions later. The $p+1$ coordinates of the $\mathrm{D} p$-brane worldvolume are denoted by $x_{\|}$, and have dimension of length. The radial coordinate $U$ has dimensions of mass, and from the $\mathrm{D} p$-branes worldvolume field theory point of view it plays the role of an energy scale. Large values of $U$ correspond to the UV regime and small values correspond to the IR regime of the Yang-Mills theory. The angular coordinates of the $(8-p)$-dimensional sphere are denoted by $\Omega_{8-p}$. They are dimensionless in the above notation. The field $\phi$ is the ten-dimensional dilaton.

We will be mostly interested in the nonconformal cases $p \neq 3$. In these cases the metric (1) has the isometry group $I S O(1, p) \times S O(9-p)$. From the Yang-Mills theory point of view $\operatorname{ISO}(1, p)$ is the Poincare symmetry while $S O(9-p)$ is the $R$-symmetry group. The Yang-Mills coupling is dimensionful, $\left[g_{Y M}^{2}\right]=[L]^{p-3}$, and we can define a dimensionless expansion parameter of the system:

$$
g_{\text {eff }}^{2}=g_{Y M}^{2} N U^{p-3}=\lambda U^{p-3} .
$$

The Yang-Mills theory is strongly coupled when $g_{\text {eff }}>1$.

The scalar curvature of the metric (1) is given by

$$
l_{s}^{2} \mathcal{R} \sim \frac{1}{g_{\text {eff }}} .
$$

We see from relation (3) that the curvature expansion in the dimensionless parameter $l_{s}^{2} \mathcal{R}$ on the supergravity side corresponds to a strong coupling expansion of the gauge theory in the inverse of the effective Yang-Mills bare coupling. It is a good expansion when $l_{s}^{2} \mathcal{R} \ll 1$, i.e., in the regime where the Yang-Mills field theory is strongly coupled. When $g_{\text {eff }}^{2} \ll 1$ we have a large curvature. In this regime we cannot trust the supergravity description. However, the Yang-Mills perturbation theory in $g_{\text {eff }}$ is valid in this regime. The overlap regime is when $g_{\text {eff }} \sim 1$.

The effective string coupling $e^{\phi}$ in Eqs. (1) can be expressed in terms of $g_{\text {eff }}$ as

$$
e^{\phi} \sim \frac{g_{\text {eff }}^{(7-p) / 2}}{N} .
$$

We see from Eq. (4) that the string loop expansion corresponds to the $1 / N$ expansion of the Yang-Mills theory. This expansion is good when the effective string coupling is small $e^{\phi} \ll 1$.

Thus, the regime where the supergravity description of the system given by Eq. (1) is valid is dictated by the conditions that both the curvature (3) and effective string coupling (4) are small. This implies that

$$
1 \ll g_{e f f}^{2} \ll N^{4 /(7-p)} .
$$

We will work in this regime. Moreover, we will consider the cases $p<6$. When $p \geqslant 6$ the above field theory limit is no longer a decoupling limit, and the brane worldvolume theory does not decouple from the bulk physics. This can be seen, for instance, by the fact that the eleven-dimensional Planck length $l_{11}^{3}=g_{s} l_{s}^{3}$ is fixed in the limit. Therefore, gravity does not decouple in eleven dimensions, which provides the proper description of the system in the UV [5]. Another way is to compute the absorption cross section for graviton scattering on the branes and see that it does not vanish in the limit [8]. From the brane-world scenario we will see that gravity and gauge fields cannot be localized.

\section{B. Stress-energy tensor correlators}

Let us now use the dual supergravity description in order to compute the two-point function of the stress-energy tensor of the Yang-Mills field theory on the $\mathrm{D} p$-branes worldvolume. Unlike the conformal case $p=3$, the two-point function changes as we vary the length scale $|x| \equiv U^{-1}$. The regime of validity of the supergravity computation is given by relation (5). As noted above this is a strong coupling regime of the gauge theory. The results that we will find will match, up to a numerical factor, with the perturbative Yang-Mills expectation in the crossover regime.

The basic idea of the correspondence between string theory and gauge theory is the identification, in the supergravity approximation, of the generating functional of the connected Green's functions in the gauge theory with the minimum of the supergravity action subject to certain boundary conditions $[2,3]$. For each operator on the Yang-Mills side we identify a field on the supergravity (string) side. The bulk field that couples to the stress-energy tensor of the gauge theory is the graviton. In the computation of the generating functional for the stress-energy tensor correlators we need to solve the graviton field equation in the background (1) subject to some boundary conditions and insert it in the supergravity action. One decomposes the metric

$$
g_{\mu \nu}=\bar{g}_{\mu \nu}+h_{\mu \nu}, \quad \mu, \nu=0, \ldots, 9
$$

where $\bar{g}_{\mu \nu}$ denotes the background metric (1) and $h_{\mu \nu}$ denotes the perturbation. The stress-energy tensor $T_{\mu \nu}$ of the brane worldvolume field theory couples to the bulk graviton as

$$
S_{i n t} \sim \int d^{p+1} x T^{\mu \nu} h_{\mu \nu}
$$


We can decompose the graviton field as

$$
h_{\mu \nu}(x, U, \Omega)=\epsilon_{\mu \nu} e^{i k \cdot x} h(U) Y\left(\Omega_{8-p}\right),
$$

where $\epsilon_{\mu \nu}$ is a constant polarization tensor, and $x$ are the $p$ +1 worldvolume coordinates. The graviton is polarized along the brane worldvolume coordinates, i.e., $\epsilon_{\mu \nu}$ $=0, \mu, \nu=p+1, \ldots, 9$. We use the transverse traceless gauge, i.e., $\partial_{\mu} h^{\mu \nu}=0$ and $h_{\mu}^{\mu}=0$, and consider the $s$-wave mode of the graviton, i.e., it does not vary along the directions of the $(8-p)$-dimensional sphere. We expand to quadratic order in the supergravity action for the graviton. The type-II supergravity equation for the graviton modes $h(U)$ is related to a minimally coupled scalar field [8]. More precisely, for certain polarization and momentum vectors the graviton equation is the minimal scalar equation

$$
f^{\prime \prime}(U)+\frac{8-p}{U} f^{\prime}(U)-k^{2} \lambda U^{p-7} f(U)=0 .
$$

In the following we will suppress the index structure of the stress-energy tensor.

We consider Eq. (9) in the range $0 \leqslant U \leqslant U_{0}$. This equation is solved by Bessel functions and we choose the regular solution that is normalized to one at $U=U_{0}$. Substituting the solution into the supergravity action, the generating functional is obtained as a pure boundary term. We construct the "flux factor" $\mathcal{F}_{T}$,

$$
\mathcal{F}_{T}=\frac{N^{2}}{\lambda^{2}}\left[f(U) U^{8-p} \partial_{U} f(U)\right]_{0}^{U_{0}},
$$

which yields the momentum-space two-point function, and we get that

$$
\langle T(k) T(-k)\rangle=\mathcal{F}_{T} .
$$

By choosing the $U_{0}$ independent nonanalytic term in $\mathcal{F}_{T}$ and Fourier transforming, we obtain the two-point function in $x$-space. When $p<5$ we get

$$
\begin{gathered}
\langle T(x) T(0)\rangle=\frac{N^{2}}{\lambda^{(2-\alpha)}} \frac{1}{|x|^{2 \alpha+p+1}}=\frac{N^{\alpha}}{g_{Y M}^{2(2-\alpha)}} \frac{1}{|x|^{2 \alpha+p+1}}, \\
\alpha=\frac{7-p}{5-p} .
\end{gathered}
$$

For $p=5$ the two-point function reads

$$
\langle T(x) T(0)\rangle=\frac{N^{2}}{\lambda^{19 / 4}} \frac{1}{|x|^{5 / 2}} e^{-|x| / \sqrt{\lambda}},
$$

where we have only written the leading large $|x|$ behavior. We have summarized the results in Table I.

Two cases that will be of particular importance for the brane-world scenarios are $p=4$ and $p=5$. It is therefore of interest to give some more details on the computation in these two cases.
TABLE I. The supergravity results for the $N$ D $p$-branes $\langle T(x) T(0)\rangle$ correlator.

\begin{tabular}{cc}
\hline \hline$p$ & $\langle T(x) T(0)\rangle$ \\
\hline 0 & $\frac{N^{7 / 5}}{g_{Y M}^{6 / 5}} \frac{1}{|x|^{19 / 5}}$ \\
1 & $\frac{N^{3 / 2}}{g_{Y M}} \frac{1}{|x|^{5}}$ \\
2 & $\frac{N^{5 / 3}}{g_{Y M}^{2 / 3}} \frac{1}{|x|^{19 / 3}}$ \\
3 & $\frac{N^{2}}{|x|^{8}}$ \\
4 & $\frac{N^{3} g_{Y M}^{2}}{|x|^{11}}$ \\
& $\frac{1}{\lambda^{19 / 4}} e^{-|x|^{5 / 2}}$ \\
\hline \hline
\end{tabular}

\section{D4-branes}

The minimal scalar equation (9) reads

$$
f^{\prime \prime}(U)+\frac{4}{U} f^{\prime}(U)-k^{2} \lambda U^{-3} f(U)=0
$$

Define the coordinate $z=2(\lambda / U)^{1 / 2}$, which has dimensions of length. The regular normalizable solution of Eq. (14) is given by

$$
f(z)=(k z)^{3} K_{3}(k z)
$$

where $K_{3}(k z)$ is the modified Bessel function. The relevant nonanalytic piece of the "flux factor" (10) reads

$$
\mathcal{F}_{T}=N^{2} \lambda k^{6} \log (k)
$$

The Fourier transform of Eq. (16) yields the result

$$
\langle T(x) T(0)\rangle=\frac{N^{3} g_{Y M}^{2}}{|x|^{11}} .
$$

At the crossover regime between the supergravity description and the perturbative Yang-Mills description $g_{Y M}^{2} N|x|^{-1} \simeq 1$ we have the matching to the expected $U(N)$ gauge theory result with $N^{2}$ degrees of freedom:

$$
\langle T(x) T(0)\rangle=\frac{N^{2}}{|x|^{10}} .
$$

The matching agrees up to a numerical factor that is independent of the rank of the gauge group $N$ and the Yang-Mills coupling $g_{Y M}$. 


\section{D5-branes}

The minimal scalar equation (9) reads

$$
f^{\prime \prime}(U)+\frac{3}{U} f^{\prime}(U)-k^{2} \lambda U^{-2} f(U)=0 .
$$

Define $U \lambda^{1 / 2}=\exp \left(z \lambda^{-1 / 2}\right)$, where $z$ has dimensions of length. The solutions of Eq. (19) are given by

$$
f(z)=\exp \left[-z \lambda^{-1 / 2}\left(1 \pm \sqrt{1+\lambda k^{2}}\right)\right]
$$

After the normalization the relevant nonanalytic piece of the "flux factor" (10) reads

$$
\mathcal{F}_{T}=\frac{N^{2}}{\lambda^{3}} \sqrt{1+\lambda k^{2}} \text {. }
$$

Fourier transforming Eq. (21) yields Eq. (13).

At the crossover regime between the supergravity description and the perturbative Yang-Mills description $g_{Y M}^{2} N|x|^{-2} \simeq 1$ we have the matching to the expected $U(N)$ gauge theory result with $N^{2}$ degrees of freedom:

$$
\langle T(x) T(0)\rangle=\frac{N^{2}}{|x|^{12}} .
$$

As before, the matching agrees up to a numerical factor that is independent of the rank of the gauge group $N$ and the Yang-Mills coupling $g_{Y M}$.

At a length scale $|x| \ll g_{Y M} / \sqrt{N}$ the effective string coupling is large and the weakly coupled description is given by the S-dual NS5-brane background. The minimally coupled scalar equation in this background is again (19) and the result (13) is obtained again.

\section{Dp-branes}

For general $p$ the correlator $\langle T(x) T(0)\rangle$ as given in Table I, matches at the crossover region between the supergravity description and the perturbative Yang-Mills description $g_{Y M}^{2} N|x|^{p-3} \simeq 1$ with the expected Yang-Mills result

$$
\langle T(x) T(0)\rangle=\frac{N^{2}}{|x|^{2(p+1)}} .
$$

We note that the result in Table I for the stress-energy twopoint function when $p=1$ was obtained in Ref. [9].

\section{Current-current correlators}

Global symmetry currents of the brane worldvolume field theory $J_{\mu}$ couple to massless bulk gauge fields $A^{\mu}$ as

$$
S_{i n t} \sim \int d^{p+1} x J_{\mu} A^{\mu} .
$$

We may distinguish two types of such gauge fields. One type of field arises from the isometries of the space on which we reduce, in our case, the $(8-p)$-dimensional sphere in Eqs. (1). These couple to $S O(9-p)$ R-symmetry currents of the
$\mathrm{D} p$-branes gauge theory. We can have additional gauge fields that couple to other global symmetry currents as, for instance, in the five-dimensional fixed points with $E_{n}$ global symmetry analyzed in Ref. [10]. We will consider the former type in the following.

In order to compute the current correlators we need to solve the graviton equation for the components of the graviton with one index along the brane worldvolume and one index along the $(8-p)$-dimensional sphere. Alternatively we can first reduce the supergravity metric (1) on the $(8-p)$-dimensional sphere and solve the $(p+2)$-dimensional gauge field equation. We will pursue the second procedure since it will also shed light later on braneworld scenarios based on various domain-wall solutions in the literature.

The dimensional reduction of the $\mathrm{D} p$-brane supergravity solutions along the sphere have been performed in Refs. $[11,12]$ using another coordinate system. We will repeat the same procedure here, except that we will use the coordinates of the supergravity solution (1). It is useful to retain the $U$ coordinate because it has the interpretation of an energy scale of the brane worldvolume field theory.

The dimensional reduction is performed by using the ansatz

$$
d s_{E}^{2}=l_{s}^{2}\left(e^{a \varphi} d s_{p+2}^{2}+e^{-p /(8-p) a \varphi} d \Omega_{8-p}^{2}\right),
$$

where $\varphi$ is a scalar field and $d s_{E}^{2}$ is the ten-dimensional $\mathrm{D} p$-branes metric written in the Einstein frame, i.e., it is related to the string frame metric (1) by $d s_{E}^{2}=e^{-\phi / 2} d s^{2}$. The constant $a$ is given by

$$
a=\frac{1}{2}\left(\frac{8-p}{p}\right)^{1 / 2}
$$

By comparing the coefficient of the $d \Omega_{8-p}^{2}$ term of the two metrics $d s_{E}^{2}$ in Eq. (25) and $d s^{2}$ in Eqs. (1) we can read the form of the scalar $\varphi$

$$
e^{\varphi}=\left[\frac{1}{N} \lambda^{(3-p) / 4} U^{-(3-p)^{2} / 4}\right]^{[(8-p) / p]^{1 / 2}} .
$$

Substituting this expression into Eq. (25) we obtain the ( $p$ +2)-dimensional metric

$$
d s_{p+2}^{2}=N^{4 / p} \lambda^{-3 / p} U^{9 / p-1}\left(d x_{\|}^{2}+\lambda U^{p-7} d U^{2}\right) .
$$

The $(p+2)$-dimensional gauge field bulk action is then given by

$$
\begin{aligned}
S_{\text {gauge }} & =\int d^{p+2} x \sqrt{g_{p+2}} e^{-[4 / \sqrt{p(8-p)}] \varphi} F^{2} \\
& \equiv \int d^{p+2} x \sqrt{g_{p+2}} \frac{1}{g_{\text {bulk }}^{2}} F^{2},
\end{aligned}
$$

where

$$
g_{\text {bulk }}^{2}=N^{-4 / p} \lambda^{3 / p-1} U^{-(3-p)^{2} / p} .
$$


This is of importance since while the gravitational and scalar fields in the reduced action are canonical in $(p+2)$ dimensions this is not the case for the gauge field as we see from Eq. (29). Note that for our purposes we omit the group theory factors and we write the action (29) schematically. In the following we will suppress the Lorentz index on the current

In order to solve the $(p+2)$-dimensional Maxwell equation, we write the $(p+2)$-dimensional gauge field as

$$
A_{\mu}(x, U)=\varepsilon_{\mu} e^{i k \cdot x} f(U) .
$$

Imposing the gauge conditions $\partial^{\mu} A_{\mu}=0, \mu=0, \ldots, p+1$, and $A_{U}=0$ we obtain

$$
f^{\prime \prime}(U)+\frac{3}{U} f^{\prime}(U)-k^{2} \lambda U^{p-7} f(U)=0 .
$$

As before, we consider Eq. (32) in the range $0 \leqslant U \leqslant U_{0}$. We solve this equation and choose a regular solution that is normalized to one at $U=U_{0}$. The generating functional for the current correlators is obtained as a pure boundary term. Again we construct the "flux factor" $\mathcal{F}_{A}$

$$
\mathcal{F}_{A}=\frac{N^{2}}{\lambda}\left[f(U) U^{3} \partial_{U} f(U)\right]_{0}^{U_{0}}
$$

and we have that

$$
\langle J(k) J(-k)\rangle=\mathcal{F}_{A} .
$$

By choosing the $U_{0}$ independent nonanalytic term in $\mathcal{F}_{A}$, and Fourier transforming we obtain for $p<5$

$$
\begin{gathered}
\langle J(x) J(0)\rangle=\frac{N^{2}}{\lambda^{(2-\alpha)}} \frac{1}{|x|^{2 \alpha+p-1}}=\frac{N^{\alpha}}{g_{Y M}^{2(2-\alpha)}} \frac{1}{|x|^{2 \alpha+p-1}}, \\
\alpha=\frac{7-p}{5-p},
\end{gathered}
$$

and for $p=5$

$$
\langle J(x) J(0)\rangle=\frac{N^{2}}{\lambda^{15 / 4}} \frac{1}{|x|^{5 / 2}} e^{-x / \sqrt{\lambda}} .
$$

The results are summarized in Table II. Consider in detail the $p=4$ and $p=5$ cases.

\section{D4-branes}

Equation (32) reads

$$
f^{\prime \prime}(U)+\frac{3}{U} f^{\prime}(U)-k^{2} \lambda U^{-3} f(U)=0 .
$$

As before, define $z=2(\lambda / U)^{1 / 2}$, which has dimensions of length. The regular normalized solution of Eq. (37) is given by

$$
f(z)=(k z)^{2} K_{2}(k z),
$$

TABLE II. The supergravity results for the $N \mathrm{D} p$-branes $\langle J(x) J(0)\rangle$ correlator.

\begin{tabular}{cc}
\hline \hline$p$ & $\langle J(x) J(0)\rangle$ \\
\hline 0 & $\frac{N^{7 / 5}}{g_{Y M}^{6 / 5}} \frac{1}{|x|^{9 / 5}}$ \\
1 & $\frac{N^{3 / 2}}{g_{Y M}} \frac{1}{|x|^{3}}$ \\
2 & $\frac{N^{5 / 3}}{g_{Y M}^{2 / 3}} \frac{1}{|x|^{13 / 3}}$ \\
3 & $\frac{N^{2}}{|x|^{6}}$ \\
4 & $\frac{N^{3} g_{Y M}^{2}}{|x|^{9}}$ \\
\hline & $\lambda^{15 / 4} \frac{1}{|x|^{5 / 2}} e^{-|x| / \sqrt{\lambda}}$ \\
\hline \hline
\end{tabular}

where $K_{2}(k z)$ is the modified Bessel function. The relevant piece of the "flux factor" (10) reads

$$
\mathcal{F}_{A}=N^{2} \lambda k^{4} \log (k)
$$

Fourier transforming Eq. (39) yields

$$
\langle J(x) J(0)\rangle=\frac{N^{3} g_{Y M}^{2}}{|x|^{9}} .
$$

At the crossover region between the supergravity description and the perturbative Yang-Mills description $g_{Y M}^{2} N|x|^{-1} \simeq 1$ we have the matching to the expected gauge theory result

$$
\langle J(x) J(0)\rangle=\frac{N^{2}}{|x|^{8}} .
$$

The matching is up to a numerical factor that is independent of the rank of the gauge group $N$ and the Yang-Mills coupling $g_{Y M}$. We have omitted the group theory factors and $\mathrm{R}$ charges as well.

\section{D5-branes}

Equation (32) is identical to the minimal scalar equation (19). We consider the same solutions (20) and construct the "flux factor" which now reads

$$
\mathcal{F}_{A}=\frac{N^{2}}{\lambda^{2}} \sqrt{1+\lambda k^{2}} .
$$

Fourier transforming Eq. (42) yields (36). 
At the crossover region between the supergravity description and the perturbative Yang-Mills description $g_{Y M}^{2} N|x|^{-2} \simeq 1$ we have the matching to the expected gauge theory result:

$$
\langle J(x) J(0)\rangle=\frac{N^{2}}{|x|^{10}} .
$$

As before, the matching is up to a numerical factor that is independent of the rank of the gauge group $N$ and the YangMills coupling $g_{Y M}$.

Similarly as for the stress-energy tensor the result (36) is also valid for large string coupling. This is because the S-dual supergravity background of $N$ NS-5 branes leads to the same equation of motion as (19).

\section{Dp-branes}

Note that the correlator $\langle J(x) J(0)\rangle$ for general $p$, as given in Table II, matches at the crossover region between the supergravity description and the perturbative Yang-Mills description $g_{Y M}^{2} N|x|^{p-3} \simeq 1$ with the expected Yang-Mills result

$$
\langle J(x) J(0)\rangle=\frac{N^{2}}{|x|^{2 p}} .
$$

Note also that, up to numerical constants that are independent of $g_{Y M}$ and $N$, the two-point functions of the stress tensor $T$ and the current $J$ are related for $p<5$ by

$$
\langle J(x) J(0)\rangle=|x|^{2}\langle T(x) T(0)\rangle
$$

and for $p=5$ by

$$
\langle J(x) J(0)\rangle=\lambda\langle T(x) T(0)\rangle .
$$

\section{BRANE-WORLDS}

We use the notation brane-world for a brane (not necessarily a $\mathrm{D} p$-brane) located at $U=U_{*}$. When $p \neq 3$ and the brane is a $\mathrm{D} p$-brane, the theory on the brane-world is described by a $(p+1)$-dimensional nonconformal super YangMills theory coupled to gravity with a cutoff $\Lambda=U_{*}$. In general, the brane-world can have extra degrees of freedom.

In this section we will analyze these brane-world scenarios with and without additional compactification of brane worldvolume coordinates. These brane-world scenarios are sometimes called dilatonic domain walls. To make the connection we note that in $p+2$ dimensions the $\mathrm{RR}$ $(p+2)$-form field $F_{p+2}=d A_{p+1}$ can be dualized and replaced by a cosmological constant. The $(p+2)$-dimensional supergravity action reduced from ten dimensions has domain wall solutions. Some of these supersymmetric solutions are the ten-dimensional $\mathrm{D} p$-branes solutions reduced on the (8 $-p$ )-dimensional sphere $[13,14,12]$.

A further reduction to be discussed is the compactification of some of the $\mathrm{D} p$-branes worldvolume coordinates. In particular the compactification of one coordinate of the D4brane worldvolume on the circle, $\mathrm{S}^{1}$ or two of the D5-brane

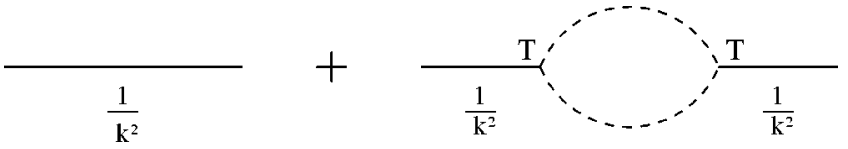

FIG. 1. The graviton propagator $G_{\text {graviton }}(k)$, where the first part is the free graviton propagation while the second part is the leading non-CFT correction.

worldvolume on a torus, $S^{1} \times S^{1}$ leads to a four-dimensional brane-world scenarios. We will discuss in more detail the relation to the brane-world scenarios based on the dilatonic domain wall solutions in the discussion section.

\section{A. Newton's law}

The $(p+1)$-dimensional gravitational coupling $\kappa_{p+1}$ is defined in terms of the ten-dimensional gravitational coupling $\kappa_{10}$ via

$$
\frac{1}{\kappa_{10}^{2}} \int d^{10} x \sqrt{g_{10}} e^{-2 \phi} \mathcal{R}_{10}=\frac{1}{\kappa_{p+1}^{2}} \int d^{p+1} x \sqrt{g_{p+1}} \mathcal{R}_{p+1} .
$$

The left-hand side (LHS) is the ten-dimensional gravitational action in the string frame metric (1) while the right-hand side (RHS) is the $(p+1)$-dimensional gravitational action in the Einstein frame. Using Eqs. (1) and (47) we obtain

$$
G_{p+1} \equiv \kappa_{p+1}^{2}=\frac{g_{Y M}^{2}}{N U_{*}^{2}},
$$

where $U_{*}$ is the UV cutoff. Note that when $U_{*} \rightarrow \infty$, the gravitational coupling (48) vanishes and gravity decouples from the brane, as expected.

Consider the correction to Newton's law in the braneworld scenarios.

\section{D4-branes}

Consider a brane-world scenario described by a fivedimensional SYM theory coupled to gravity with a cutoff at $U=U_{*}$. The correction to the Newton's law can be computed from the graviton propagator $G_{\text {graviton }}(k)$ [15], as in Fig. 1. The first part is the free graviton propagation while the second part is the leading non-CFT correction

$$
\begin{aligned}
G_{\text {graviton }}(k) & \sim \frac{1}{k^{2}}+\frac{1}{k^{2}} \kappa_{5}\langle T(k) T(-k)\rangle \kappa_{5} \frac{1}{k^{2}} \\
& =\frac{1}{k^{2}}\left[1+N^{3} g_{Y M}^{2} G_{5} k^{4} \log (k)\right],
\end{aligned}
$$

where we have used Eq. (16), and $G_{5}$ is given by Eq. (48). Fourier transforming in five dimensions yield

$$
G_{\text {graviton }}(x) \sim \frac{1}{x^{3}}\left(1+N^{3} g_{Y M}^{2} \frac{G_{5}}{x^{4}}\right) .
$$


From relation (50) we can read the modification to the fivedimensional Newton's force law $1 / r^{3}$ :

$$
F_{\text {Newton }}=\frac{G_{5} m_{1} m_{2}}{r^{3}}\left(1+\frac{N^{2} \lambda G_{5}}{r^{4}}\right) .
$$

\section{D5-branes}

Consider a six-dimensional brane-world scenario. The correction to the Newton's law can be computed as before from the graviton propagator $G_{\text {graviton }}(k)$ as in Fig. 1. Again, the first part is the free graviton propagation while the second part is the leading non-CFT correction. Using Eq. (21) we get

$$
G_{\text {graviton }}(k) \sim \frac{1}{k^{2}}\left(1+\frac{N^{2} G_{6}}{\lambda^{3}} \frac{\sqrt{1+\lambda k^{2}}}{k^{2}}\right),
$$

where $G_{6}$ is given by Eq. (48). Fourier transforming in six dimensions yields at large $x$

$$
G_{\text {graviton }}(x) \sim \frac{1}{x^{4}}\left[1+c \frac{N^{2} G_{6}}{\lambda^{2}}\left(\frac{x}{\sqrt{\lambda}}\right)^{3 / 2} e^{-x / \sqrt{\lambda}}\right]
$$

where by $c$ we denote a numerical constant which we neglected. The modification to the six-dimensional Newton's force law $1 / r^{4}$ reads

$$
F_{\text {Newton }}=\frac{G_{6} m_{1} m_{2}}{r^{4}}\left[1+c \frac{N^{2} G_{6}}{\lambda^{2}}\left(\frac{r}{\sqrt{\lambda}}\right)^{3 / 2} e^{-r / \sqrt{\lambda}}\right]
$$

\section{Dimensional reduction}

In order to arrive at four-dimensional brane-world scenarios one can further compactify the worldvolume coordinates. In the D4-brane case we compactify one coordinate on a circle while in the D5-brane case we compactify two coordinates on a torus. Consider first the D4-brane scenario where we compactify on a circle of radius $R$. At distances $|x|=U^{-1}$ in the regime

$$
R \ll|x| \ll g_{Y M}^{2} N
$$

we can neglect the momenta along the circle direction and we are still in the supergravity regime defined by relation (5). Thus, Fourier transforming in four dimensions, the gravitational force is then

$$
F_{\text {Newton }}=\frac{G_{4} m_{1} m_{2}}{r^{2}}\left(1+\frac{N^{2} \lambda G_{4} R}{r^{4}}\right)
$$

where $G_{4} R=G_{5}$.

Consider next the D5-brane scenario where we compactify on two circles of radius $R$. At distances in the regime

$$
R \ll|x| \ll\left(g_{Y M}^{2} N\right)^{1 / 2},
$$

we can neglect the momenta along the circle direction and we are still in the supergravity regime defined by relation (5). Fourier transforming in four dimensions, the gravitational force reads

$$
F_{\text {Newton }}=\frac{G_{4} m_{1} m_{2}}{r^{2}}\left[1+c \frac{N^{2} G_{4} R^{2}}{\lambda^{2}}\left(\frac{r}{\sqrt{\lambda}}\right)^{-1 / 2} e^{-r / \sqrt{\lambda}}\right],
$$

where $G_{4} R^{2}=G_{6}$.

\section{Discussion}

In the following we compare the gravitational force based on the nonconformal brane-world scenarios with the gravitational force in brane-world scenarios based on $\mathrm{AdS}_{d+1}$,

$$
F_{\text {Newton }}=\frac{G_{d} m_{1} m_{2}}{r^{d-2}}\left(1+\frac{c_{d} G_{d}}{r^{d-2}}\right)
$$

where $c_{d}$ is the central charge of the underlying conformal field theory. Comparing the above result with Eq. (51) in five dimensions, Eq. (54) in six dimensions and Eqs. (56) and (58) in four dimensions after dimensional reduction, we see that in the nonconformal brane-world scenarios the corrections to Newton's law have different $r$ dependences. In particular, the D5-brane geometry leads to corrections that are exponentially suppressed. As we will see later, this corresponds to the fact that the Kaluza-Klein spectrum has a mass gap. Experimental measurements of Newton's law can distinguish between these different brane-world scenarios.

\section{B. Coulomb's law}

The effective $(p+1)$-dimensional gauge coupling, $g_{\text {brane }}$, on the $p$-brane can be obtained via

$$
\int d^{p+2} x \sqrt{g_{p+2}} \frac{1}{g_{\text {bulk }}^{2}} F_{(p+2)}^{2}=\int d^{p+1} x \frac{1}{g_{\text {brane }}^{2}} F_{(p+1)}^{2},
$$

where $F_{(p+2)}$ and $F_{(p+1)}$ denote the field strength of the gauge field in $p+2$ and $p+1$ dimensions, respectively. The LHS is the $(p+2)$-dimensional gauge field action after the dimensional reduction (25) while the RHS is the effective $(p+1)$-dimensional gauge field action. Using Eq. (30) we obtain for $p>3$

$$
g_{\text {brane }}^{2}=\frac{1}{N^{2} U_{*}^{p-3}},
$$

where $U_{*}$ is the UV cutoff.

Consider the correction to Coulomb's law in these braneworld scenarios.

\section{D4-branes}

Consider a five-dimensional brane-world scenario. The correction to the Coulomb's law can be computed from the propagator for a vector field $G_{v e c t o r}(k)$ as in Fig. 2. The first 


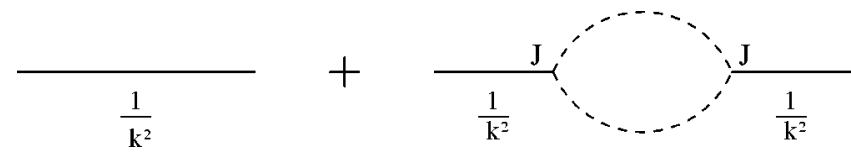

FIG. 2. The vector field propagator $G_{\text {vector }}(k)$ where the first part is the free vector propagation while the second part is the leading non-CFT correction.

part is the free vector propagation, while the second part is the leading non-CFT correction

$$
\begin{aligned}
G_{\text {vector }}(k) & \sim \frac{1}{k^{2}}+\frac{1}{k^{2}} g_{5}\langle J(k) J(-k)\rangle g_{5} \frac{1}{k^{2}} \\
& =\frac{1}{k^{2}}\left[1+N^{3} g_{Y M}^{2} g_{5}^{2} k^{2} \log (k)\right],
\end{aligned}
$$

where we have used Eq. (39). The gauge coupling $g_{5}$ is given by Eq. (61) for $p=4$.

Fourier transforming in five dimensions yields

$$
G_{\text {vector }}(x) \sim \frac{1}{x^{3}}\left(1+N^{3} g_{Y M}^{2} \frac{g_{5}^{2}}{x^{2}}\right) \text {. }
$$

The modification to the five-dimensional Coulomb's force law $1 / r^{3}$ reads

$$
F_{\text {Coulomb }}=\frac{g_{5}^{2} q_{1} q_{2}}{r^{3}}\left(1+\frac{N^{2} \lambda g_{5}^{2}}{r^{2}}\right)
$$

\section{D5-branes}

Consider a six-dimensional brane-world scenario. The correction to the Coulomb's law can be computed as before from the vector propagator $G_{v e c t o r}(k)$ as in Fig. 2. Again, the first part is the free graviton propagation while the second part is the leading non-CFT correction. Using Eq. (42) we obtain

$$
G_{\text {vector }}(k) \sim \frac{1}{k^{2}}\left(1+\frac{N^{2} g_{6}^{2}}{\lambda^{2}} \frac{\sqrt{1+\lambda k^{2}}}{k^{2}}\right),
$$

where $g_{6}$ is given by Eq. (61) for $p=5$. The modification to the six-dimensional Coulomb's force law $1 / r^{4}$ reads

$$
F_{\text {Coulomb }}=\frac{g_{6}^{2} q_{1} q_{2}}{r^{4}}\left[1+c \frac{N^{2} g_{6}^{2}}{\lambda}\left(\frac{r}{\sqrt{\lambda}}\right)^{3 / 2} e^{-r / \sqrt{\lambda}}\right]
$$

\section{Dimensional reduction}

Again, in order to arrive at four-dimensional brane-world scenarios one can further compactify the worldvolume coordinates. In the D4-brane case we compactify one coordinate on a circle while in the D5-brane case we compactify two coordinates on a torus. Consider first the D4-branes scenario where we compactify on a circle of radius $R$. At distances satisfying regime (55) the Coulomb force reads

$$
F_{\text {Coulomb }}=\frac{g_{4}^{2} q_{1} q_{2}}{r^{2}}\left(1+\frac{N^{2} \lambda g_{4}^{2} R}{r^{2}}\right)
$$

where $g_{4}^{2} R=g_{5}^{2}$.

Consider next the D5-branes scenario where we compactify on two circles of radius $R$. At distances satisfying regime (57) we get

$$
F_{\text {Coulomb }}=\frac{g_{4}^{2} q_{1} q_{2}}{r^{2}}\left[1+c \frac{N^{2} g_{4}^{2} R^{2}}{\lambda}\left(\frac{r}{\sqrt{\lambda}}\right)^{-1 / 2} e^{-r / \sqrt{\lambda}}\right] .
$$

where $g_{4}^{2} R^{2}=g_{6}^{2}$.

\section{Discussion}

In the following we compare the Coulomb force based on the nonconformal brane-world scenarios with the Coulomb force in brane-world scenarios based on $\mathrm{AdS}_{d+1}$,

$$
F_{\text {Coulomb }}=\frac{g_{d}^{2} q_{1} q_{2}}{r^{d-2}}\left(1+\frac{g_{d}^{2} a_{d}}{r^{d-4}}\right)
$$

where $a_{d}$ is the coefficient of the current-current correlator of the underlying conformal field theory. Note that for $d=4$ we cannot use this formula and the corrections are logarithmic.

Comparing the above result with Eq. (64) in five dimensions, Eq. (66) in six dimensions and Eqs. (67),(68) in four dimensions after dimensional reduction, we see that in the nonconformal brane-world scenarios the corrections to Coulomb's law have different $r$ dependences. In particular, the D5-brane geometry leads to corrections that are exponentially suppressed. As we will see later, this corresponds to the fact that the Kaluza-Klein spectrum has a mass gap. Again, experimental measurements of Coulomb's law can distinguish between these different brane-world scenarios.

\section{Graviton and gauge field localization}

In the previous calculation of the Newton and Coulomb laws, a massless mode with behavior $1 / k^{2}$ was assumed to be localized on the brane worldvolume. However, one can explicitly check the localization properties of the bulk graviton and gauge fields on the $p$-brane located at $U=U_{*}$ by solving the equation of motion in the background geometry (1) and computing the Green's function $\mathcal{G}_{k}$.

By invoking the bulk-boundary correspondence, the bulk Green's function can be used to obtain an alternative derivation of the two-point functions $\langle\mathcal{O O}\rangle$ in the dual theory, considered in Sec. II. Since the Green's function represents the sum over all connected diagrams we obtain the relation 


$$
\langle\mathcal{O}(k) \mathcal{O}(-k)\rangle=\frac{k^{2}}{\kappa}-\mathcal{G}_{k}^{-1},
$$

where for the stress-energy tensor $T_{\mu \nu}$ we use the graviton propagator $\left(\kappa=-2 G_{p+1}\right)$, while for the vector current $J_{\mu}$ we use the gauge field propagator $\left[\kappa=(3-p) g_{p+1}^{2}\right]$. For $p$ $<6$ there always exists a term in Eq. (70) that does not depend on the cutoff $U_{*}$. The results for the stress-energy tensor and current correlator, obtained in this way, are all in agreement with those found in Sec. II.

Let us now consider the graviton and gauge field Green's function to explicitly check the localization properties on the p-brane.

\section{Graviton}

Consider a bulk graviton in the effective $(p+2)$-dimensional brane-world. The minimally coupled scalar equation (9) determines the mass and localization properties of the $(p+1)$-dimensional Kaluza-Klein modes, where $k^{2}=-m^{2}$. The differential operator (9) is self-adjoint and the solutions $f_{m}$ form a complete set that satisfies the orthonormal relation

$$
\int_{0}^{U_{*}} d U U f_{m} f_{m^{\prime}}=\delta_{m m^{\prime}}
$$

When $m=0$ we find that the equation of motion (9) always has a solution $f_{0}(U)=$ const, and there is always a normalizable mode. This is consistent with Eq. (48).

To check whether gravity is actually localized on the $p$-brane we must compute the Green's function for two sources on the brane. In order to do this the first question that needs to be addressed is the boundary conditions. This is particularly important because except for the case $p=3$, there is singular behavior at $U=0$. This singularity corresponds to the massless open string degrees of freedom that are missing in the description. We expect the singularity to be resolved once the required degrees are added, and we will continue to impose Hartle-Hawking boundary conditions at $U=0$, as in the $p=3$ case. Thus following the procedure outlined in the Appendix the expression for the scalar Green's function when $U=U^{\prime}=U_{*}$ is given by

$$
\begin{aligned}
\mathcal{G}_{k}\left(U_{*}, U_{*}\right)= & -i \frac{g_{Y M}^{4}}{\sqrt{\lambda k}} U_{*}^{(p-9) / 2} \\
& \times \frac{H_{\alpha}^{(1)}\left(i(\alpha-1) \sqrt{\lambda} k U_{*}^{(p-5) / 2}\right)}{H_{\alpha-1}^{(1)}\left(i(\alpha-1) \sqrt{\lambda} k U_{*}^{(p-5) / 2}\right)},
\end{aligned}
$$

where $H^{(1)}$ is the Hankel function of the first kind. When $p<5$ the Green's function can be written in the form $\mathcal{G}_{k}\left(U_{*}, U_{*}\right)=\mathcal{G}_{k}^{(0)}+\mathcal{G}_{k}^{(K K)}$, where the zero mode contribution $\mathcal{G}_{k}^{(0)}$ and the Kaluza-Klein contribution $\mathcal{G}_{k}^{(K K)}$ are given by

$$
\begin{aligned}
\mathcal{G}_{k}^{(0)}= & -2 \frac{G_{p+1}}{k^{2}}, \\
\mathcal{G}_{k}^{(K K)}= & i \frac{g_{Y M}^{4}}{\sqrt{\lambda} k} U_{*}^{(p-9) / 2} \\
& \times \frac{H_{\alpha-2}^{(1)}\left(i(\alpha-1) \sqrt{\lambda} k U_{*}^{(p-5) / 2}\right)}{H_{\alpha-1}^{(1)}\left(i(\alpha-1) \sqrt{\lambda} k U_{*}^{(p-5) / 2}\right)} .
\end{aligned}
$$

We see from the form of $\mathcal{G}_{k}^{(0)}$ that one obtains Newton's law on the $p$-brane located at $U_{*}$. This is consistent with the fact that the graviton zero mode is also localized there. The corresponding corrections to the $(p+1)$-dimensional Newton's law are obtained from $\mathcal{G}_{k}^{(K K)}$ and one obtains the result found earlier for the D4-brane.

When $p=5$ the Green's function has the form

$$
\mathcal{G}_{k}\left(U_{*}, U_{*}\right)=-\frac{G_{6}}{k^{2}}\left(1+\sqrt{1+\lambda k^{2}}\right),
$$

and again there is a localized massless mode $\left(1 / k^{2}\right)$ at $U$ $=U_{*}$. The corresponding corrections to Newton's law arising from the Kaluza-Klein continuum lead to exponentially suppressed corrections and agree with the result found earlier.

When $p \geqslant 6$ one finds that there is an equal and opposite contribution arising from $\mathcal{G}_{k}^{(K K)}$ that precisely cancels the $\mathcal{G}_{k}^{(0)}$ contribution. The remaining leading contribution from $\mathcal{G}_{k}^{(K K)}$ does not lead to the usual Newton's law. Thus for $p$ $\geqslant 6$ we do not obtain Newton's law and there is no localized mode in the brane-world with $1 / k^{2}$ behavior.

\section{Gauge fields}

The equation of motion for the $(p+1)$-dimensional Kaluza-Klein gauge fields is governed by Eq. (32) where $k^{2}=-m^{2}$. The differential operator in Eq. (32) is self-adjoint and the solutions $f_{m}$ form a complete set that satisfies the orthonormal relation

$$
\int_{0}^{U_{*}} d U U^{p-4} f_{m} f_{m^{\prime}}=\delta_{m m^{\prime}} .
$$

Notice that when $m=0$ there is always a constant solution to Eq. (32). Thus only for $p \geqslant 4$ will the zero mode be normalizable. This is consistent with Eq. (61).

Analogous to the graviton case we can obtain the Green's function for two charged (under the gauge field) sources localized on the $p$-brane. Following the procedure outlined in the Appendix one obtains the gauge field Green's function

$$
\begin{aligned}
\mathcal{G}_{k}\left(U_{*}, U_{*}\right)= & -i \frac{\sqrt{\lambda}}{N^{2} k} U_{*}^{(1-p) / 2} \\
& \times \frac{H_{\alpha-1}^{(1)}\left(i(\alpha-1) \sqrt{\lambda} k U_{*}^{(p-5) / 2}\right)}{H_{\alpha-2}^{(1)}\left(i(\alpha-1) \sqrt{\lambda} k U_{*}^{(p-5) / 2}\right)} \\
\equiv & \mathcal{G}_{k}^{(0)}\left(U_{*}\right)+\mathcal{G}_{k}^{(K K)}\left(U_{*}\right),
\end{aligned}
$$

where for $p \neq 5$ we have 


$$
\begin{aligned}
\mathcal{G}_{k}^{(0)}= & (3-p) \frac{g_{p+1}^{2}}{k^{2}}, \\
\mathcal{G}_{k}^{(K K)}= & i \frac{\sqrt{\lambda}}{N^{2} k} U_{*}^{(1-p) / 2} \\
& \times \frac{H_{\alpha-3}^{(1)}\left(i(\alpha-1) \sqrt{\lambda} k U_{*}^{(p-5) / 2}\right)}{H_{\alpha-2}^{(1)}\left(i(\alpha-1) \sqrt{\lambda} k U_{*}^{(p-5) / 2}\right)},
\end{aligned}
$$

and $g_{p+1}=1 /\left(N U_{*}^{(p-3) / 2}\right)$ is the effective gauge coupling for $p>3$ on the $p$-brane. When $p<3$, the leading contribution $\mathcal{G}_{k}^{(0)}$ is positive and cancels against an equal and opposite contribution from $\mathcal{G}_{k}^{(K K)}$. Thus, there is no localized mode with $1 / k^{2}$ behavior.

For $p=3$, the zero mode contribution $\mathcal{G}_{k}^{(0)}$ vanishes and the leading Kaluza-Klein contribution is

$$
\mathcal{G}_{k}^{(K K)}=\frac{1}{N^{2} \log (k)} \frac{1}{k^{2}} \equiv \frac{e_{e f f}^{2}(k)}{k^{2}} .
$$

As noticed in [16-18], even though the bulk zero mode is nonnormalizable, one can still think of a 4D gauge field with an effective gauge coupling that runs logarithmically to zero in the infrared.

When $p=4$ the zero mode contribution $\mathcal{G}_{k}^{(0)}$ is nonvanishing and there is a localized gauge field on the brane. The corrections to Coulomb's law arise from the Kaluza-Klein continuum and agree with the result obtained earlier for the D4-brane.

The D5-brane case is similar to that encountered for the minimally coupled scalar. The Green's function is now

$$
\mathcal{G}_{k}\left(U_{*}, U_{*}\right)=-\frac{g_{6}^{2}}{k^{2}}\left(1+\sqrt{1+\lambda k^{2}}\right),
$$

and one obtains the six-dimensional Coulomb's law. These results are identical to the graviton case except for the couplings and again we see the mass gap in the Kaluza-Klein spectrum of the gauge field.

When $p>5$ the contribution $\mathcal{G}_{k}^{(0)}$ is again canceled by an equal and opposite contribution arising from $\mathcal{G}_{k}^{(K K)}$. Thus there is no localized gauge field on the $p$-brane with $1 / k^{2}$ behavior.

\section{DISCUSSION}

In the following we will discuss in more detail the relation between our work and the brane-world scenarios based on dilatonic domain wall solutions. Consider domain wall solutions in $D$ dimensions which are solutions of the field equations derived from the action

$$
S=\int d^{D} x \sqrt{g}\left[\mathcal{R}-\frac{1}{2}(\partial \varphi)^{2}-2 \Lambda e^{b \varphi}\right]+\int d^{D-1} x \mathcal{L}
$$

The field $\varphi$ is a $D$-dimensional scalar field, $\Lambda$ is a bulk cosmological constant and $b$ is a constant parametrized in terms of a quantity $\Delta$ :

$$
b^{2}=\Delta+\frac{2(D-1)}{(D-2)} \text {. }
$$

The Lagrangian $\mathcal{L}$ is a delta-function source added to the bulk action. It provides a cutoff for the boundary of the bulk solution.

When $b=0$ one obtains AdS solutions where the dilaton is constant and the dual description is some CFT. More precisely, if we use only a slice of AdS, the description of the brane-world scenario is in terms of a CFT coupled to gravity with a cutoff. For example, when $D=5$ then $\Delta_{\mathrm{AdS}_{5}}=-8 / 3$, and one obtains the solution [6]. This can originate, for instance, from a ten-dimensional $\mathrm{AdS}_{5} \times \mathrm{S}^{5}$ solution of typeIIB supergravity, with an $\mathcal{N}=4$ SCFT dual description. In the truncated brane-world version, the dual conformal field theory is modified by introducing an ultraviolet cutoff, and gauging the Poincare symmetry $[18,19]$.

Similarly, when $D=6$ one obtains a six-dimensional AdS solution [20], where $\Delta_{\mathrm{AdS}_{6}}=-5 / 2$. This can arise as a reduction of a ten-dimensional warped $\mathrm{AdS}_{6}$ solution corresponding to the D4-D8 brane system [10]. The dual field theory is a five-dimensional CFT. A five-dimensional domain wall solution can be obtained by compactifying one of the brane dimensions on a circle $S^{1}$ and analyzed in terms of the corresponding dual theory in Ref. [21]. Other similar solutions were obtained in Ref. [22].

Domain wall solutions can also be constructed where $b$ $\neq 0$, and the dilaton is no longer constant. One example is in $D=5$ and $\Delta=-12 / 5$ with a varying dilaton $[13,14,11,12]$, which can be obtained from the near-horizon metric of D4branes compactified on $\mathrm{S}^{1} \times \mathrm{S}^{4}$. Following the results in Sec. III A, the Newtonian law obtained from the dimensional reduction of the D4-brane solution is consistent with the corrections obtained in Ref. [12]. Our derivation indicates that the dual theory of this domain wall solution is the fivedimensional SYM theory compactified on $\mathrm{S}^{1}$. In comparison to the Randall-Sundrum scenario where the correction to the Newton potential is proportional to $1 / r^{3}$, here the corrections are $1 / r^{5}$. We also considered the gauge fields in this domain wall background. Gauge fields that arise from the isometries of $\mathrm{S}^{4}$ have a zero mode localized on the domain wall. The corrections to the Coulomb law arising from the KaluzaKlein continuum are given by Eq. (64), and are related to the current correlators of the dual field theory.

Another $D=5$ solution, with $\Delta=-2$, arises from the near-horizon metric of D5-branes compactified on $\mathrm{T}^{2} \times \mathrm{S}^{3}$. This case, analyzed in Sec. III A, gives rise to a Newtonian force with exponentially suppressed corrections (see also Ref. [12]). Our analysis indicates that the dual field theory is a six-dimensional SYM compactified on $\mathrm{T}^{2}$. The exponentially suppressed corrections have two equivalent interpretations. In the supergravity description, they arise because the Kaluza-Klein continuum is separated from the zero mode by a mass gap. In the six-dimensional SYM theory they corre- 
spond to a mass gap of the theory developed in the strongly coupled regime. As before, we can study the gauge fields in the domain wall background. Those that arise from the isometries of $S^{3}$ have a zero mode localized on the domain wall. The Coulomb law corrections are exponentially suppressed, and they are related to the current correlators of the sixdimensional SYM theory.

\section{ACKNOWLEDGMENTS}

The work of T.G. is supported by the FNRS, contract No. 21-55560.98.

\section{APPENDIX: GREEN'S FUNCTION IN THE EXTREMAL D $p$-BRANE GEOMETRY}

We will follow the derivation of the Green's function presented in Ref. [23], except that we will consider the extremal $\mathrm{D} p$-brane geometry. Let us first consider the case of the minimally coupled scalar $\phi$ in ten dimensions. Introducing a source function $\mathcal{J}$, one obtains

$$
\partial_{M}\left(\sqrt{-g} g^{M N} \partial_{N} \phi(X)\right)=\sqrt{-g} \mathcal{J}(X) .
$$

where $X=\left(x, U, \theta_{i}\right)$, with $x$ denoting the $p+1$ worldvolume coordinates. The corresponding Green's function for Eq. (A1) can then be defined as

$$
\phi(X)=\int d^{10} X^{\prime} \sqrt{-g} G\left(X ; X^{\prime}\right) \mathcal{J}\left(X^{\prime}\right) .
$$

If we now consider the Fourier transform of the Green's function

$$
G\left(X ; X^{\prime}\right)=\sum_{\left\{n_{i}\right\}} e^{i\left\{n_{i}\right\} \theta_{i}} \int \frac{d^{p+1} k}{(2 \pi)^{p+1}} e^{i k \cdot\left(x-x^{\prime}\right)} \mathcal{G}_{k}\left(U, U^{\prime}\right),
$$

where the discrete Fourier transform is over the hyperspherical coordinates of $\mathrm{S}^{8-p}$, then the Fourier component $\mathcal{G}_{k}\left(U, U^{\prime}\right)$ must satisfy the equation

$$
\begin{aligned}
& {\left[\partial_{u}\left(U^{8-p} \partial_{u}\right)-\lambda k^{2} U+m_{\theta_{i}}^{2} U^{6-p}\right] \mathcal{G}_{k}\left(U, U^{\prime}\right)} \\
& \quad=g_{Y M}^{4} \delta\left(U-U^{\prime}\right) .
\end{aligned}
$$

The standard procedure for solving Eq. (A4) is to use the solution to the homogeneous equation in the regions $U$ $<U^{\prime}$ and $U>U^{\prime}$, and then impose matching conditions at $U=U^{\prime}$. If we now restrict to the case where $m_{\theta_{i}}^{2}=0$ and write

$$
\mathcal{G}_{k}\left(U, U^{\prime}\right)=\theta\left(U-U^{\prime}\right) \mathcal{G}_{>}+\theta\left(U^{\prime}-U\right) \mathcal{G}_{<},
$$

the solution to the homogeneous equation for $U>U^{\prime}$ and $p$ $\neq 5$ is given by

$$
\begin{aligned}
\mathcal{G}_{>}\left(U, U^{\prime}\right)= & i A_{>}\left(U^{\prime}\right) U^{(p-7) / 2}\left[J_{\alpha-1}\left(q_{*}\right) H_{\alpha}^{(1)}(q)\right. \\
& \left.-H_{\alpha-1}^{(1)}\left(q_{*}\right) J_{\alpha}(q)\right],
\end{aligned}
$$

with $\alpha=(7-p) /(5-p)$ and where we have imposed the Neumann condition $\left.\partial_{u} \mathcal{G}_{k}\left(U, U^{\prime}\right)\right|_{U=U_{*}}=0$. We have also defined $q=i(\alpha-1) \sqrt{\lambda} k U^{(p-5) / 2}$, and $H_{\alpha}^{(1)}$ is the Hankel function of the first kind of order $\alpha$. As noted earlier there is a singularity at $U=0$, but since the solution can be made finite there we will continue to impose the Hartle-Hawking boundary condition. Thus for $U<U^{\prime}$ we obtain

$$
\mathcal{G}_{<}\left(U, U^{\prime}\right)=i A_{<}\left(U^{\prime}\right) U^{(p-7) / 2} H_{\alpha}^{(1)}(q) .
$$

The unknown functions $A_{<}\left(U^{\prime}\right)$ and $A_{>}\left(U^{\prime}\right)$ are determined by imposing matching conditions at $U=U^{\prime}$. Continuity of $\mathcal{G}_{k}$ at $U=U^{\prime}$ leads to the condition

$$
\left.\mathcal{G}_{>}\right|_{U=U^{\prime}}=\left.\mathcal{G}_{<}\right|_{U=U^{\prime}},
$$

while the discontinuity in $\partial_{u} \mathcal{G}_{k}$ gives the condition

$$
\left.\left(\partial_{u} \mathcal{G}_{>}-\partial_{u} \mathcal{G}_{<}\right)\right|_{U=U^{\prime}}=g_{Y M}^{4} U^{\prime p-6} .
$$

This leads to the solutions

$$
\begin{aligned}
A_{<}\left(U^{\prime}\right)= & \frac{\pi g_{Y M}^{4}}{5-p} U^{\prime(p-7) / 2} \frac{H_{\alpha}^{(1)}\left(q^{\prime}\right)}{H_{\alpha-1}^{(1)}\left(q_{*}\right)}, \\
A_{>}\left(U^{\prime}\right)= & i \frac{\pi g_{Y M}^{4}}{5-p} U^{\prime}(p-7) / 2\left[\frac{J_{\alpha-1}\left(q_{*}\right)}{H_{\alpha-1}^{(1)}\left(q_{*}\right)}\right. \\
& \left.\times H_{\alpha}^{(1)}\left(q^{\prime}\right)-J_{\alpha}\left(q^{\prime}\right)\right] .
\end{aligned}
$$

Finally, substituting these functions into the equations for $\mathcal{G}_{>}$ and $\mathcal{G}_{<}$gives the expression for the Green's function in the extremal $\mathrm{D} p$-brane geometry

$$
\begin{aligned}
\mathcal{G}_{k}\left(U, U^{\prime}\right)= & i \frac{\pi g_{Y M}^{4}}{5-p}\left(U U^{\prime}\right)^{(p-7) / 2} \frac{H_{\alpha}^{(1)}\left(q_{<}\right)}{H_{\alpha-1}^{(1)}\left(q_{*}\right)} \\
& \times\left[J_{\alpha-1}\left(q_{*}\right) H_{\alpha}^{(1)}\left(q_{>}\right)-H_{\alpha-1}^{(1)}\left(q_{*}\right) J_{\alpha}\left(q_{>}\right)\right],
\end{aligned}
$$

where in $q_{>}\left(q_{<}\right)$we have defined $U_{>}\left(U_{<}\right)$to be the greater (lesser) of $U$ and $U^{\prime}$. The Green's function (A12) is the general expression when $p \neq 5$ for a minimally coupled scalar in the extremal $\mathrm{D} p$-brane geometry. Notice also that from the pole condition $H_{\alpha-1}^{(1)}\left(2 i \sqrt{\lambda} k /(5-p) U_{*}^{(p-5) / 2}\right)=0$, there is a branch cut singularity at $k=0$. This represents the Kaluza-Klein continuum beginning at $m=0$, where $k^{2}=$ $-m^{2}$. When we restrict the coordinates to the location of the $p$-brane at $U=U^{\prime}=U_{*}$ we obtain the expression (72).

When $p=5$ the solutions to the homogeneous equation are no longer Bessel functions (20). Imposing the same boundary conditions as for $p \neq 5$ gives the Green's function 


$$
\begin{aligned}
\mathcal{G}_{k}\left(U, U^{\prime}\right)= & -\frac{g_{Y M}^{2}}{N} \frac{1+\gamma}{2 \gamma k^{2}} \frac{1}{U U^{\prime}}\left(\frac{U_{*}}{U_{>}}\right)^{-\gamma}\left[(\gamma-1)\left(\frac{U_{*}}{U_{<}}\right)^{\gamma}\right. \\
& \left.+(\gamma+1)\left(\frac{U_{*}}{U_{<}}\right)^{-\gamma}\right],
\end{aligned}
$$

where $\gamma=\sqrt{1+\lambda k^{2}}$. When $U=U^{\prime}=U_{*}$ we recover the expression (75). Notice also that from the pole condition $k^{2}$ $=0$ there is a massless mode. In addition from the pole condition $\gamma=0$, there is also a branch cut singularity beginning at $m=1 / \sqrt{\lambda}$. Thus when $p=5$, the Kaluza-Klein continuum is separated from the zero mode by a mass gap $1 / \sqrt{\lambda}$.

The Green's function for the gauge field can be obtained by following a similar procedure to the minimally coupled scalar. In the case of $p \neq 5$ the expression is

$$
\begin{aligned}
\mathcal{G}_{k}\left(U, U^{\prime}\right)= & i \frac{\pi g_{Y M}^{2}}{N(5-p)} \frac{1}{U U^{\prime}} \frac{H_{\alpha-1}^{(1)}\left(q_{<}\right)}{H_{\alpha-2}^{(1)}\left(q_{*}\right)} \\
& \times\left[J_{\alpha-2}\left(q_{*}\right) H_{\alpha-1}^{(1)}\left(q_{>}\right)\right. \\
& \left.-H_{\alpha-2}^{(1)}\left(q_{*}\right) J_{\alpha-1}\left(q_{>}\right)\right],
\end{aligned}
$$

while for $p=5$ it is simply

$$
\begin{aligned}
\mathcal{G}_{k}\left(U, U^{\prime}\right)= & -\frac{1}{N^{2}} \frac{1+\gamma}{2 \gamma k^{2}} \frac{1}{U U^{\prime}}\left(\frac{U_{*}}{U_{>}}\right)^{-\gamma}\left[(\gamma-1)\left(\frac{U_{*}}{U_{<}}\right)^{\gamma}\right. \\
& \left.+(\gamma+1)\left(\frac{U_{*}}{U_{<}}\right)^{-\gamma}\right] .
\end{aligned}
$$

Again we see that the $(p+1)$-dimensional Kaluza-Klein spectrum has the same characteristics as that found for the minimally coupled scalar. When $U=U^{\prime}=U_{*}$ we obtain the results (77) for $p \neq 5$, and (81) for $p=5$.
[1] J. Maldacena, Adv. Theor. Math. Phys. 2, 231 (1998).

[2] S. S. Gubser, I. R. Klebanov, and A. M. Polyakov, Phys. Lett. B 428, 105 (1998).

[3] E. Witten, Adv. Theor. Math. Phys. 2, 253 (1998).

[4] O. Aharony, S. S. Gubser, J. Maldacena, H. Ooguri, and Y. Oz, Phys. Rep. 323, 183 (2000).

[5] N. Itzhaki, J. M. Maldacena, J. Sonnenschein, and S. Yankielowicz, Phys. Rev. D 58, 046004 (1998).

[6] L. Randall and R. Sundrum, Phys. Rev. Lett. 83, 4690 (1999).

[7] H. Verlinde, Nucl. Phys. B580, 264 (2000).

[8] M. Alishahiha, H. Ita, and Y. Oz, J. High Energy Phys. 06, 002 (2000).

[9] A. Hashimoto and N. Itzhaki, Phys. Lett. B 454, 235 (1999).

[10] A. Brandhuber and Y. Oz, Phys. Lett. B 460, 307 (1999).

[11] D. Youm, Nucl. Phys. B576, 106 (2000); B576, 139 (2000); B589, 315 (2000); B596, 289 (2001).

[12] M. Cvetic, H. Lu, and C. N. Pope, Phys. Rev. D 63, 086004 (2001).

[13] H. J. Boonstra, K. Skenderis, and P. K. Townsend, J. High Energy Phys. 01, 003 (1999).
[14] K. Behrndt, E. Bergshoeff, R. Halbersma, and J. P. van der Schaar, Class. Quantum Grav. 16, 3517 (1999).

[15] J. Maldacena (unpublished); E. Witten (unpublished); S. S. Gubser, Phys. Rev. D 63, 084017 (2001).

[16] A. Pomarol, Phys. Lett. B 486, 153 (2000); A. Pomarol, Phys. Rev. Lett. 85, 4004 (2000).

[17] N. Kaloper, E. Silverstein, and L. Susskind, J. High Energy Phys. 05, 031 (2001).

[18] N. Arkani-Hamed, M. Porrati, and L. Randall, J. High Energy Phys. 08, 017 (2001).

[19] R. Rattazzi and A. Zaffaroni, J. High Energy Phys. 04, 021 (2001).

[20] T. Gherghetta and M. Shaposhnikov, Phys. Rev. Lett. 85, 240 (2000).

[21] E. Ponton and E. Poppitz, J. High Energy Phys. 02, 042 (2001).

[22] T. Gherghetta, E. Roessl, and M. Shaposhnikov, Phys. Lett. B 491, 353 (2001); T. Gherghetta, hep-th/0010276.

[23] S. B. Giddings, E. Katz, and L. Randall, J. High Energy Phys. 03, 023 (2000). 